\title{
DECREASING CHAINS WITHOUT LOWER BOUNDS IN THE RUDIN-FROLÍK ORDER
}

\author{
EVA BUTKOVIČOVÁ
}

(Communicated by Dennis Burke)

\begin{abstract}
We prove that for every uncountable cardinal less than continuum there exists a chain in the Rudin-Frolik order of $\beta \omega-\omega$ isomorphic to the inverse order of this cardinal and without a lower bound.
\end{abstract}

\section{INTRODUCTION}

In [vD] E. K. van Douwen constructed a sequence $\left\langle D_{\beta}: \beta<\mathfrak{c}\right\rangle$ of countable discrete subsets of $\beta \omega$ such that

$$
\begin{gathered}
(\forall \epsilon, \eta<\mathfrak{c})\left(\epsilon<\eta \longrightarrow D_{\eta} \subseteq \bar{D}_{\epsilon}-D_{\epsilon}\right), \\
\left|\bigcap_{\beta<\mathfrak{c}} \bar{D}_{\beta}\right|=1 .
\end{gathered}
$$

He asked whether for every cardinal $\mu<\mathfrak{c}$ of uncountable cofinality such sequences exist of length $\mu$. The question is closely related to the Rudin-Frolik order $\leq_{R F}$ of ultrafilters on $\omega$. One readily sees that if $\left\langle D_{\beta}: \beta<\mu\right\rangle$ is such a sequence and $p$ is the point in the intersection $\bigcap_{\beta<\mu} \bar{D}_{\beta}$ then $\left\langle\Omega\left(D_{\beta}, p\right): \beta<\right.$ $\mu\rangle$ is a strictly decreasing $\leq_{R F}$-chain in $\beta \omega$ without lower bound. The chains bound, the aim of this paper is to prove that such chains exist; we prove

(1.3) Theorem. For every cardinal $\mu$ between $\omega$ and $\mathfrak{c}$ there exists a strictly decreasing $\leq_{R F}$-chain $\left\langle p_{\beta}: \beta<\mu\right\rangle$ without an $\leq_{R F}$-lower bound.

Let us note that this shows that such a chain exists whenever $\omega \leq \mu \leq \mathfrak{c}$ : in [BB] such a sequence was constructed for $\mu=\omega$, and of course van Douwen's construction mentioned above gives such a sequence for $\mu=\mathfrak{c}$.

Received by the editors September 16,1985 and, in revised forms, March 25, 1989 and July 20, 1989. The results of this paper have been presented to the Colloquim on Topology in Pe'cs (Hungary), August 1989.

1980 Mathematics Subject Classification (1985 Revision). Primary 54A25, 04A20.

Key words and phrases. Ultrafilter, Rudin-Frolik order, independent family. 


\section{DEFINITIONS AND PRELIMINARIES}

We shall use standard set-theoretical notation.

For an arbitrary set $I$, we put $[I]^{<\omega}=\{A \subseteq I:|A|<\omega\}$. For $\mathscr{F} \subseteq \mathscr{P}(\omega)$ we put

$$
\left\langle\langle\mathscr{F}\rangle=\left\{A \subseteq \omega: \exists H \in[\mathscr{F}]^{<\omega}, \bigcap H \subseteq A\right\} .\right.
$$

Thus, if $\mathscr{F}$ is centered then $\langle\langle\mathscr{F}\rangle\rangle$ is the filter generated by $\mathscr{F}$, otherwise $\langle\langle\mathscr{F}\rangle\rangle=\mathscr{P}(\omega)$.

The Fréchet filter on $\omega$ is denoted by $F$. Ultrafilters are assumed to be on $\omega$. We reserve the capitals $X$ and $Y$ for sets of ultrafilters. When we say "countable" we mean "countably infinite". If $I$ is a set then $\mathrm{Fn}(I, \omega)$ denotes the set of finite partial functions from $I$ to $\omega$. We let $\mathfrak{c}$ denote the cardinality of the continuum.

(2.3.1) For each $\xi$ the family $\left\{A_{\xi, n}: n \in \omega\right\}$ is a partition of $\omega$.

(2.3.2) For every $H \in \mathscr{F}$ and every $\varphi \in \mathrm{Fn}(I, \omega)$ the intersection $A(\varphi) \cap H$ is nonempty. Here

$$
A(\varphi)=\bigcap_{\xi \in \operatorname{dom} \varphi} A_{\xi, \varphi(\xi)} .
$$

There exists an independent family $\left\{A_{\xi, n}: \xi \in \mathfrak{c}, n \in \omega\right\}$ with respect to the filter $F$, see for example [K] or [EKa]. An easy example for topologists: identify $\omega$ with a countable dense subset $D$ of the product ' $\omega$, and put $A_{\xi, n}=$ $\{f \in D: f(\xi)=n\}$.

Independent families are used in inductive constructions, they help to make sure that the induction in question can be carried out till the end. The construction given in this paper hopefully shows how useful independent families can be. Some basic properties that will be useful are:

If $\left\{A_{\xi, n}: \xi \in I, n \in \omega\right\}$ is independent with respect to $\mathscr{F}$, if $\varphi \in \operatorname{Fn}(I, \omega)$, and if $M \supseteq A(\varphi)$ then the family $\left\{A_{\xi, n}: \xi \in\right.$ $I-\operatorname{dom} \varphi, n \in \omega\}$ is independent with respect to $\langle\langle\mathscr{F} \cup\{M\}\rangle$.

If $\left\{A_{\xi, n}: \xi \in I, n \in \omega\right\}$ is independent with respect to $\mathscr{F}$ then $\mathscr{F}$ is not an ultrafilter because for every $n$ and every $\xi$ both $A_{\xi, n} \notin \mathscr{F}$ and $\omega-A_{\xi, n} \notin \mathscr{F}$.

Now we turn to the definition of the Rudin-Frolík order $\leq_{R F}$ of nontrivial ultrafilters on $\omega$. 
Let $p, q \in \beta \omega-\omega$. We say $p \leq_{R F} q$ if there exists a discrete set $X=\left\{x_{n}: n<\omega\right\}$ in $\beta \omega$ such that

$$
A \in q \equiv\left\{n: A \in x_{n}\right\} \in p \text {. }
$$

The ultrafilter $q$ is denoted by $\Sigma(X, p)$ and $p$ is denoted by $\Omega(X, q)$. Let us note that they generally depend on the indexing of $X$.

The relation $\leq_{R F}$ is reflexive and transitive but not antisymmetric. The best one say is that if $p \leq_{R F} q$ and $q \leq_{R F} p$ then $p$ and $q$ are type equivalent, which means that there is a permutation $\pi$ of $\omega$ such that $\pi(p)=q$.

We say $p<_{R F} q$ if $p \leq_{R F} q$ but not $q \leq_{R F} p$. It is straightforward to show that:

$$
\begin{aligned}
& p<_{R F} q \text { iff there is a discrete set } X \subseteq \beta \omega-\omega \text { such that } \\
& q=\Sigma(X, p) .
\end{aligned}
$$

Hence an ultrafilter $q$ has an $\leq_{R F}$-predecessor iff there is a countable discrete set $X \subseteq \beta \omega-\omega$ such that $q \in \bar{X}-X$.

We mention some technical properties of $\leq_{R F}$. It is not too hard to show that if $X$ and $Y$ are countable subsets of $\beta \omega$ and $\bar{X} \cap Y=X \cap \bar{Y}=\varnothing$ then $\bar{X} \cap \bar{Y}=\varnothing$. It follows that

[F2] If $p \in \bar{X} \cap \bar{Y}$ then either $p \in \overline{X \cap \bar{Y}-Y}$, in which case $\Omega(X, p)<_{R F} \Omega(Y, q)$, or $p \in \overline{Y \cap \bar{X}-X}$, in which case $\Omega(Y, p)$ $<_{R F} \Omega(X, q)$, or $p \in \overline{X \cap Y}$, in which case $\Omega(X, p)$ and $\Omega(Y, p)$ are type equivalent.

An important consequence is that the $\leq_{R F}$-predecessors of a point in $\beta \omega-\omega$ are linearly ordered. Another useful fact is the following:

If $p \in \beta \omega-\omega$ and if $X$ and $Y$ are countable discrete sets of ultrafilters then $\Sigma(X, p)<_{R F} \Sigma(Y, p)$ iff $\left\{n: x_{n}<_{R F} y_{n}\right\} \in p$.

\section{Plan of the proof of Theorem 1.3}

Fix a cardinal $\mu$ between $\omega$ and $\mathfrak{c}$.

First we deal with the case that $\mu$ has countable cofinality. Consider the sequence $\left\langle X_{\alpha}: \alpha<\mathfrak{c}\right\rangle$ constructed by van Douwen. It is not too hard to show that each point in $\bigcup_{r<c} X_{\alpha}$ may be chosen to have character $c$. Fix a strictly increasing sequence $\left\langle\mu_{n}: n<\omega\right\rangle$ cofinal in $\mu$. Now the proof from [BB] can be used to find a point $p$ in $\bigcap_{n<\omega} \bar{X}_{\mu_{n}}$ such that $\left\langle\Omega\left(X_{\mu_{n}}, p\right): n<\omega\right\rangle$ has no $\leq_{R F}$-lower bound, but then neither has $\left\langle\Omega\left(X_{\beta}, p\right): \beta<\mu\right\rangle$.

The case when $\mu$ has uncountable cofinality requires more work. It is evident from (2.8) that we need a sequence $\left\langle X_{\beta}: \beta<\mu\right\rangle$ of countable discrete sets and a point $p$ in $\beta \omega-\omega$ satifying:

$$
p \in \bigcap_{\beta<\mu} \bar{X}_{\beta}
$$

and

$$
\text { if } \gamma<\beta<\mu \text { then } X_{\beta} \subseteq \bar{X}_{\eta}-X_{\eta} \text {. }
$$


The sequence $\left\langle\Omega\left(X_{\beta}, p\right): \beta<\mu\right\rangle$ will then be strictly decreasing with respect to $\leq_{R F}$. A necessary and sufficient condition that it has no $\leq_{R F}$-lower bound is formulated in the following lemma, where we let $K=\bigcap_{\beta<\mu} \bar{X}_{\beta}$.

Lemma. The sequence $\left\langle\Omega\left(X_{\beta}, p\right): \beta<\mu\right\rangle$ has no $\leq_{R F}$-lower bound if and only if for every countable and discrete set $D \subseteq K-\{p\}$ we have $p \notin \bar{D}$.

Proof. We establish the contrapositive.

If $p \in \bar{Y}-Y$ for some countable and discrete $Y \subseteq K$ then by (2.8) $\Omega(Y, p)<_{R F} \Omega\left(X_{\beta}, p\right)$ for all $\beta<\mu$.

On the other hand if $Y$ is countable and discrete and $\Omega(Y, p)<_{R F} \Omega\left(X_{\beta}, p\right)$ for all $\beta$ then, again by (2.8), we must have $p \in \overline{Y \cap \bar{X}_{\beta}-X_{\beta}}$ for all $\beta$. As $Y$ is countable and the cofinality of $\mu$ is uncountable, we have $Y \cap K=Y \cap \bar{X}_{\beta}$ for some $\beta$. It follows that $p \in \overline{Y \cap K}-Y$.

This gives us the third condition that has to be met:

(3.3) for every countable and discrete $Y \subseteq K-\{p\}$ we have $p \notin \bar{Y}$.

The aim of the rest of the paper is to construct $p$ and $\left\langle X_{\beta}: \beta<\mu\right\rangle$ satisfying (3.1), (3.2), and (3.3).

\section{Plan of the construction}

We shall construct by induction on $\alpha<\mathfrak{c}$ filters $\mathscr{F}^{\text {(r }}$ and $\mathscr{F}_{\beta, n}^{\prime r}$ for $\beta<\mu$ and $n<\omega$. In the end we shall let

$$
p=\bigcup_{r<c} \mathscr{F}^{(r} \quad \text { and } \quad x_{\beta, n}=\bigcup_{\kappa<c} \mathscr{F}_{\beta, n}^{\alpha} .
$$

Let us investigate what conditions will have to be met in order that $p$ and the $x_{\beta, n}$ satisfy (3.1), (3.2), and (3.3). To abbreviate some formulas we will put $\mathscr{F}_{\mu, n}^{i n}=\mathscr{F}^{(x}$ for $n<\omega$.

First of all, to ensure that $p$ and the $x_{\beta, n}$ are filters we want

(o) If $\alpha<\alpha^{\prime}<\mathfrak{c}$ then $\mathscr{F}_{\beta, n}^{\prime \prime} \subseteq \mathscr{F}_{\beta, n}^{\alpha^{\prime}}$ for $\beta \leq \mu$ and $n<\omega$.

To make sure that in the end we will have ultrafilters we let $\left\{M_{\alpha}: \alpha<\right.$ c, $\alpha$ odd $\}$ enumerate $\mathscr{P}(\omega)$ and we will have

(i) If $\alpha$ is odd then for every $\beta \leq \mu$ and $n<\omega$

$$
M_{c} \in \mathscr{F}_{\beta, n}^{\alpha+1} \text { or } \omega-M_{\alpha} \in \mathscr{F}_{\beta, n}^{\alpha+1} .
$$

If we write $x_{\mu, n}=p$ for $n<\omega$ then we can combine (3.1) and (3.2) into (3.1a) if $\gamma<\beta \leq \mu$ then $X_{\beta} \subseteq \bar{X}_{\gamma}-X_{\gamma}$.

To ensure this we need for every $\alpha$

(ii) If $\gamma<\beta \leq \mu, n<\omega$, and $A \in \mathscr{F}_{\beta, n}^{(\alpha)}$ then $E(A, \gamma)=\left\{i: A \in \mathscr{F}_{\gamma, i}^{\left({ }_{1}\right.}\right\}$ is infinite, and

(iii) If $\gamma<\beta \leq \mu$ and $i<\omega$ then there is an $A \in \mathscr{F}^{\prime \prime}, i$ such that $\omega-A \in$ $\mathscr{F}_{\beta, n}^{\prime r}$ for every $n$. 
Note that (ii) has the effect of putting $\mathscr{F}_{\beta, n}^{\alpha}$ in the closure of $\left\{\mathscr{F}_{\gamma, i}^{\alpha}: i<\omega\right\}$ and that (iii) ensures that $\mathscr{F}^{\alpha}{ }^{k}$ is not in the closure of $\left\{\mathscr{F}_{\beta, n}{ }^{k}: n<\omega\right\}$ for any $i$. Also note that once (iii) is satisfied for some $\alpha$, it is also satisfied for all later $\alpha^{\prime}$, because of (o). In fact we shall take care of (iii) right at the beginning, when $\alpha=0$.

Unfortunately a construction like this may break down prematurely. For example if $\mathscr{F}^{\text {k }}$ would be an ultrafilter there would be no way of guaranteeing (vii) below anymore. To prevent this and other problems we use two independent families, $\mathscr{A}=\left\{A_{\eta, n}: \eta<\mathfrak{c}, n<\omega\right\}$ and $\mathscr{B}=\left\{B_{\eta, i}: \eta<\mathfrak{c}, i<\omega\right\}$.

During the construction we shall keep track of subsets $I_{\alpha}$ and $J_{\alpha}$ of $c$ satisfying

(iv) For every $\alpha$ both $\mathfrak{c}-I_{\alpha}$ and $\mathfrak{c}-J_{\alpha}$ have cardinality at most $|\alpha|+\mu$, moreover the sequences of the $I_{\alpha}$ 's and $J_{\alpha}$ 's are decreasing.

We shall have:

(v) the family $\mathscr{A}_{\alpha}=\left\{A_{\eta, n}: \eta \in I_{\alpha}, n<\omega\right\}$ is independent with respect to $\mathscr{F}_{\beta, n}^{k}$ for every $\beta \leq \mu$ and $n<\omega$.

The family $\mathscr{B}$ is used to keep other filters from being ultrafilters: during our construction we also construct (automatically) the ultrafilters $\Omega\left(X_{y}, x_{\beta, n}\right)$ for $\gamma<\beta \leq \mu$ and $n<\omega$. At stage $\alpha$ the filters $\mathscr{G}_{\beta, \gamma, n}^{\alpha}=\left\{E(A, \gamma): A \in \mathscr{F}_{\beta, n}^{\alpha}\right\}$ are approximations to these. We shall have:

(vi) If $\gamma<\beta \leq \mu$ and $n<\omega$ then the family $\mathscr{B}_{\alpha}=\left\{B_{\eta, i}: \eta \in J_{\alpha}, i<\omega\right\}$ is independent with respect to $\mathscr{G}_{\beta, \gamma, n}^{\alpha}$.

Note that (vi) is much stronger than (ii).

Finally we show how to take care of (3.3): let $\left\{\mathscr{D}_{\alpha}: \alpha<\mathfrak{c}, \alpha\right.$ even $\}$ enumerate the collection of all partitions of $\omega$ in such a way that each partition is listed $c$ times. We do the following:

(vii) If $\alpha$ is even and if $\mathscr{D}_{\alpha}=\left\{D_{n}: n<\omega\right\}$ is such that for every $n$ $\omega-D_{n} \in \mathscr{F}^{k}$ and for every $\gamma<\mu$ the set $\left\{i: D_{n} \in \mathscr{F}^{{ }^{\prime}{ }^{\prime}, i}\right.$ is infinite then do as follows:

For $\gamma<\mu$ let $L_{\gamma}=\left\{i: \exists n D_{n} \in \mathscr{F}_{\gamma, i}^{\alpha}\right\}$. There are two possibilities:

(1) The family $\mathscr{B}_{\alpha}$ is independent with respect to $\left\langle\left\langle\mathscr{G}_{\mu, \gamma, 0}^{\alpha} \cup\left\{L_{\gamma}\right\}\right\rangle\right\rangle$ for every $\gamma$. In this case we partition $D_{n}$ into $n+1$ infinite pieces $D_{n, 0} \cdots D_{n, n}$ and we make sure that for every $f \in{ }^{\omega} \omega$ with $f(n) \leq n$ for all $n$ the set $Z_{f}=\omega-\bigcup_{n<\omega} D_{n, f(n)}$ is in $\mathscr{F}^{(x+1}$.

(2) There is a $\gamma$ such that $\mathscr{B}_{\alpha}$ is not independent with respect to this filter (or it is not a filter). In this case we will add $\omega-\bigcup\left\{A_{\gamma, i}: i \in L_{\gamma}\right\}$ to $\mathscr{F}^{\alpha}$, for the least such $\gamma$.

If none of the above holds we do nothing.

To see why this works suppose that in the end $Y=\left\{y_{n}: n<\omega\right\} \subseteq K-\{p\}$ is discrete. Find a partition $\mathscr{D}=\left\{D_{n}: n<\omega\right\}$ of $\omega$ such that for all $n$ $D_{n} \in y_{n}$ and $\omega-D_{n} \in p$. Next fix an (even) $\alpha$ such that $\mathscr{D}=\mathscr{D}_{r}$ and 
$\mathscr{D} \subseteq\left\{M_{\eta}: \eta<\alpha, \eta\right.$ odd $\}$. Then the assumptions of (vii) were satisfied at stage $\alpha$ (the sets $\left\{i: D_{n} \in \mathscr{F}_{\gamma, i}{ }^{\alpha}\right\}$ are all infinite because $Y \subseteq \bigcap_{\gamma<\mu} \bar{X}_{\gamma}$ ).

If (1) holds define $f$ by $f(n)=$ the $i$ for which $D_{n, i} \in y_{n}$. Then $Z_{f} \in p$ and $\omega-Z_{f} \in y_{n}$ for all $n$, so that $p \notin \bar{Y}$.

If (2) holds then one can readily check that $Y \subseteq \overline{\left\{x_{\gamma, i}: i \in L_{\gamma}\right\}}$ whereas $p$ is not in this closure.

\section{THE CONSTRUCTION}

As promised we begin by ensuring (iii) right away. We let

$$
\mathscr{F}^{0}=\left\langle\left\langle F \cup\left\{\bigcup_{j \in A} A_{\eta, j}: \eta<\mu, A \in F\right\}\right\rangle\right\rangle
$$

and for $\beta<\mu$ and $n<\omega$

$$
\begin{aligned}
\mathscr{F}_{\beta, n}^{0}=\left\langle\left\langleF \cup \left\{\bigcup_{j \in A} A_{\eta, j}: \eta\right.\right.\right. & <\beta, A \in F\} \\
& \left.\left.\cup\left\{A_{\beta, n}\right\} \cup\left\{A_{\eta, j}: n \in B_{\eta, j}, \beta<\eta<\mu\right\}\right\rangle\right\rangle .
\end{aligned}
$$

We put $I_{0}=J_{0}=\mathfrak{c}-\mu$. We only have to verify

(iii) If $\gamma<\beta$ and $i<\omega$ then $A_{\gamma, i} \in \mathscr{F}_{\gamma, i}^{0}$, but $\omega-A_{\gamma, i}=\bigcup_{j \neq i} A_{\gamma, j} \in \mathscr{F}_{\beta, n}^{0}$ for all $n$.

(vi) Note that we used "levels" from $\mathscr{A}$ when building the $\mathscr{F}_{\beta, n}^{0}$.

(vii) One can readily check that

$$
E\left(A_{\beta, n}, \gamma\right)= \begin{cases}\varnothing, & \text { if } \gamma>\beta \\ \{n\}, & \text { if } \gamma=\beta \\ B_{\beta, n}, & \text { if } \gamma<\beta\end{cases}
$$

and

so that

$$
E\left(\bigcup_{j \in A} A_{\eta, j}, \gamma\right)= \begin{cases}\omega, & \text { if } \gamma>\eta \\ A, & \text { if } \gamma=\eta \\ \bigcup_{j \in A} B_{\eta, j}, & \text { if } \gamma<\eta\end{cases}
$$

$$
\begin{aligned}
\mathscr{G}_{\beta, \gamma, n}^{0}=\left\langle\left\langleF \cup \left\{\bigcup_{j \in A} B_{\eta, j}: A\right.\right.\right. & \in F, \gamma<\eta<\beta\} \\
& \left.\left.\cup\left\{B_{\beta, n}\right\} \cup\left\{B_{\eta, j}: n \in B_{\eta, j}, \beta<\eta<\mu\right\}\right\rangle\right\rangle .
\end{aligned}
$$

It is now straightforward to check (vii).

In case $\alpha$ is a limit we simply let

$$
\mathscr{F}_{\beta, n}^{\prime \prime}=\bigcup_{\delta<r} \mathscr{F}_{\beta, n}^{\delta}, \quad I_{r}=\bigcap_{\delta<n} I_{\delta} \quad \text { and } \quad J_{\alpha}=\bigcap_{\delta<n} J_{\delta} .
$$


We leave it to the reader to verify that $\mathscr{G}_{\beta, \gamma, n}^{\alpha}=\bigcup_{\delta<\alpha} \mathscr{G}_{\beta, \gamma, n}^{\delta}$ and that (o) through (vii) hold.

Suppose that $\alpha$ is odd. We have to add $M_{\alpha}$ or $\omega-M_{\alpha}$ to each of the filters $\mathscr{F}_{\beta, n}{ }^{\alpha}$. We collect the filters to which $M_{\alpha}$ certainly cannot be added: let $C$ be the set of those pairs $\langle\beta, n\rangle$ for which $\left\langle\left\langle\mathscr{F}_{\beta, n}^{(k)} \cup\left\{M_{\alpha}\right\}\right\rangle\right\rangle$ is not a filter or, if it is, the family $\mathscr{A}_{c}$ is not independent with respect to it.

For each $\langle\beta, n\rangle$ we choose $\varphi_{\beta, n} \in \operatorname{Fn}\left(I_{\alpha}, \omega\right)$ and $H_{\beta, n} \in \mathscr{F}_{\beta, n}^{\alpha}$ such that $H_{\beta, n} \cap M_{\alpha} \cap A\left(\varphi_{\beta, n}\right)=\varnothing$, and we let $I_{\alpha+1}=I_{\alpha}-\bigcup\left\{\operatorname{dom} \varphi_{\beta, n}:\langle\beta, n\rangle \in C\right\}$. Clearly $\left|I_{\alpha}-I_{\alpha+1}\right| \leq \mu$ so that $\left|\mathfrak{c}-I_{\alpha+1}\right| \leq|\alpha+1|+\mu$.

It would be tempting to add $M_{\alpha}$ to $\mathscr{F}_{\beta, n}^{\alpha}$ if $\langle\beta, n\rangle \notin C$ and $\omega-M_{\alpha}$ to the other $\mathscr{F}_{\beta, n}^{\alpha}$. Indeed, this would create no independence problems for $\mathscr{A}_{(x+1}$. However we also have to deal with the filters $\mathscr{G}_{\beta, \gamma, n}^{(r}$ : if $M_{\alpha}$ is added to $\mathscr{F}_{\beta, n}^{(r)}$ then $E\left(M_{\alpha}, \gamma\right)$ should be well-behaved with respect to $\mathscr{B}_{\alpha+1}$ and $\mathscr{G}_{\beta, \gamma, n}^{\alpha+1}$.

For this we let $C_{0}=C$, and given $C_{\eta}$, we let $C_{\eta+1}$ be the union of $C_{\eta}$ and the set of those pairs $\langle\beta, n\rangle$ for which $\mathscr{B}_{\alpha}$ is not independent with respect to $\left\langle\left\langle G_{\beta, \gamma, n}^{\alpha} \cup\left\{\left\{k:\langle\gamma, k\rangle \notin C_{\eta}\right\}\right\}\right\rangle\right.$, if $\eta$ is a limit we let $C_{\eta}=\bigcup_{\xi<\eta} C_{\xi}$.

We claim that $C_{\omega_{1}+1}=C_{\omega_{1}}$. For suppose that $\langle\beta, n\rangle \in C_{\omega_{1}+1}-C_{\omega_{1}}$ and fix a $\gamma<\beta$ witnessing this. Take an $\eta<\omega_{1}$ such that $\langle\gamma, k\rangle \in C_{\omega_{1}}$ iff $\langle\gamma, k\rangle \in C_{\eta}$. We then find that $\langle\beta, n\rangle \in C_{\eta+1} \subseteq C_{\omega_{1}}$ anyway.

Put $\widehat{C}=C_{\omega_{1}}$ and note that by the argument given above, $\langle\beta, n\rangle \in \widehat{C}$ iff for every $\gamma<\beta, \mathscr{B}_{\alpha r}$ is independent with respect to $\left\langle\left\langle\mathscr{G}_{\beta, \gamma, n}^{\alpha r} \cup\left\{L_{\gamma}\right\}\right\rangle\right\rangle$, where $L_{\gamma}=\{k:\langle\gamma, k\rangle \notin \widehat{C}\}$.

Now we define

$$
\mathscr{F}_{\beta, n}^{\alpha+1}=\left\langle\left\langle\mathscr{F}_{\beta, n}^{\alpha} \cup\left\{\omega-M_{r r}\right\}\right\rangle\right\rangle \quad \text { if }\langle\beta, n\rangle \in \widehat{C}
$$

and

$$
\mathscr{F}_{\beta, n}^{\alpha+1}=\left\langle\left\langle\mathscr{F}_{\beta, n}^{\alpha} \cup\left\{M_{\alpha}\right\}\right\rangle\right\rangle \quad \text { if }\langle\beta, n\rangle \notin \widehat{C}
$$

An important observation at this point is that $E\left(M_{1,}, \gamma\right)=L_{r}$ and that $E\left(\omega-M_{r}, \gamma\right)=\omega-L_{\gamma}$, for every $\gamma$.

We already defined $I_{r+1}$. To define $J_{\alpha+1}$, choose for every $\langle\beta, n\rangle \in \widehat{C}$, say in $C_{\eta+1}-C_{\eta}$, a $\gamma<\beta$ witnessing this, together with $\tau_{\beta, n} \in \operatorname{Fn}\left(J_{1,}, \omega\right)$ and $G_{\beta, n} \in \mathscr{F}_{\beta, n}^{\prime \prime}$ such that $E\left(G_{\beta, n}, \gamma\right) \cap\left\{k:\langle\gamma, k\rangle \notin C_{\eta}\right\} \cap B\left(\tau_{\beta, n}\right)=\varnothing$. Now let $J_{c+1}=J_{r}-\bigcup\left\{\operatorname{dom} \tau_{\beta, n}:\langle\beta, n\rangle \in \widehat{C}-C\right\}$.

We must check (v) if $\langle\beta, n\rangle \notin \widehat{C}$ then, by the choice of $C$, the family $\mathscr{A}_{\text {or }}$ is independent with respect to $\mathscr{F}_{\beta, n}^{\alpha+1}$, hence so is the smaller family $\mathscr{A}_{1+1}$. We now show by induction on $\eta$ that if $\langle\beta, n\rangle \in C_{\eta}$ then $\mathscr{A}_{(r+1}$ is independent with respect to $\mathscr{F}_{\beta, n}^{\mathrm{a}+1}$.

If $\eta=0$ then $\stackrel{H}{\beta, n}_{n} \cap A\left(\varphi_{\beta, n}\right) \subseteq \omega-M_{\alpha}$ and $\operatorname{dom} \varphi_{\beta, n} \subseteq I_{\alpha+1}-I_{o r}$, so by $2.4 \mathscr{A}_{(r+1}$ is independent with respect to $\mathscr{F}_{\beta, n}^{(r+1}$. 
Now assume $\langle\beta, n\rangle \in C_{\eta+1}-C_{\eta}$ and find $\gamma<\beta, G_{\beta, n}$ and $\tau_{\beta, n}$ as above. Then $G_{\beta, n} \cap B\left(\tau_{\beta, n}\right) \subseteq\left\{k:\langle\gamma, k\rangle \in C_{\eta}\right\} \subseteq \omega-L_{\gamma}=E\left(\omega-M_{\alpha}, \gamma\right)$. Now let $H \in \mathscr{F}_{\beta, n}{ }^{\prime}$. By assumption $\mathscr{B}_{\alpha}$ is independent with respect to $\mathscr{G}_{\beta, \gamma, n}^{\prime r}$, so that $E(H, \gamma) \cap G_{\beta, n} \cap B\left(\tau_{\beta, n}\right) \neq \varnothing$. But then there is an $i \in \omega$ with $i \in E(H, \gamma)$ and $\langle\gamma, i\rangle \in C_{\eta}$. It follows that $H \in \mathscr{F}_{\gamma, i}^{\alpha}, \omega-M_{\alpha} \in \mathscr{F}_{\gamma, i}^{\alpha+1}$ and, since $\mathscr{A}_{\alpha+1}$ is independent with respect to $\mathscr{F}_{\gamma, i}^{(\alpha+1}, H \cap \omega-M_{\alpha} \cap A(\varphi) \neq \varnothing$ for every $\varphi \in \operatorname{Fn}\left(I_{\alpha+1}, \omega\right)$.

We verify (vi) in a similar way: if $\langle\beta, n\rangle \notin \widehat{C}$ then for $\gamma<\beta, \mathscr{G}_{\beta, \gamma, n}^{\alpha+1}=$ $\left\langle\left\langle\mathscr{G}_{\beta, \gamma, n}^{\alpha} \cup\left\{E\left(M_{\alpha}, \gamma\right)\right\}\right\rangle\right\rangle=\left\langle\left\langle\mathscr{G}_{\beta, \gamma, n}^{\alpha} \cup\left\{L_{\gamma}\right\}\right\rangle\right.$. Then $\mathscr{B}_{\alpha}$ is independent with respect to $\mathscr{G}_{\beta, \gamma, n}^{\alpha+1}$, hence so is $\mathscr{B}_{(\alpha+1}$.

If $\langle\beta, n\rangle \in C_{0}$ then $H_{\beta, n} \cap A\left(\varphi_{\beta, n}\right) \cap M_{\alpha}=\varnothing$. Hence for every $\gamma<\beta$ and $i \in E\left(H_{\beta, n}, \gamma\right)$ the family $\mathscr{A}_{\alpha \gamma}$ is not independent with respect to $\left\langle\left\langle\mathscr{F}_{\gamma^{\prime}, i} \cup\left\{M_{\alpha}\right\}\right\rangle\right.$ either. Thus, $E\left(H_{\beta, n}, \gamma\right) \subseteq \omega-L_{\gamma}=E\left(\omega-M_{\alpha}, \gamma\right)$ and, in fact, $\mathscr{G}_{\beta, \gamma, n}^{(++1}=$ $\mathscr{G}_{\beta, \gamma, n}^{\alpha}$; and we have (vi) in this case.

Finally assume that $\langle\beta, n\rangle \in C_{\eta+1}-C_{\eta}$ and consider $\gamma<\beta, G_{\beta, n} \in \mathscr{F}_{\beta, n}^{\prime \prime}$ and $\tau_{\beta, n}$ as above. We know that $E\left(G_{\beta, n}, \gamma\right) \cap B\left(\tau_{\beta, n}\right) \subseteq\left\{i:\langle\gamma, i\rangle \in C_{\eta}\right\} \subseteq$ $\omega-L_{\gamma}=E\left(\omega-M_{\alpha}, \gamma\right)$.

Now let $H \in \mathscr{F}_{\beta, n}^{\alpha}, \delta<\beta$ and $\tau \in \operatorname{Fn}\left(J_{\alpha+1}, \omega\right)$. We must show that the set $E(H, \delta) \cap E\left(\omega-M_{\alpha}, \delta\right) \cap B(\tau)$ is nonempty.

Case 1. $\delta=\gamma$. In this case the set contains $E(H, \delta) \cap E\left(G_{\beta, n}, \delta\right) \cap B\left(\tau_{\beta, n}\right) \cap$ $B(\tau)$, which is nonempty, because $\mathscr{B}_{{ }}$is independent with respect to $\mathscr{G}_{\beta, \gamma, n}^{i n}$ and $\tau \cup \tau_{\beta, n} \in \operatorname{Fn}\left(I_{\alpha}, \omega\right)$.

Case 2. $\delta<\gamma$. Fix $i \in E(H, \gamma) \cap E\left(G_{\beta, n}, \gamma\right) \cap B\left(\tau_{\beta, n}\right)$, then $\langle\gamma, i\rangle \in C_{\eta}$ and

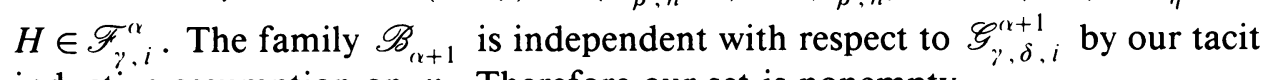
inductive assumption on $\eta$. Therefore our set is nonempty.

Case 3. $\delta>\gamma$. Clearly now $\langle\delta, l\rangle \in C_{\eta+1} \subseteq \widehat{C}$ whenever $G_{\beta, n} \in \mathscr{F}_{\delta, l}^{\prime \prime}$. Therefore $E\left(G_{\beta, n}, \delta\right) \subseteq\left\{l: \omega-M_{\alpha} \in \mathscr{F}_{\delta, l}^{(\alpha+1}\right\}$, which means that $\mathscr{G}_{\beta, \delta, n}^{\alpha+1}=$ $\mathscr{G}_{\beta, \delta, n}^{\prime \prime}$.

Suppose now that $\alpha$ is even. Assume $\mathscr{D}_{\alpha}=\left\{D_{i}: i<\omega\right\}$ satisfies the assumptions of (vii).

If (1) holds then we have to partition the sets $D_{i}$ somehow. Pick one element $\xi$ from $I_{\alpha}$ and let $I_{\alpha+1}=I_{\alpha}-\{\xi\}$. Note that, since $D_{n} \in \mathscr{F}_{\gamma, i}^{*}$ for very many $\langle\gamma, n\rangle$ and all $i$, the intersection $D_{i} \cap A_{\xi, k}$ is infinite for every $i$ and $k$. We define $D_{i, k}=D_{i} \cap A_{\xi, k}$ if $k<i$ and $D_{i, i}=D_{i}-\bigcup_{k<i} D_{i, k}$.

By (vii) we know what $\mathscr{F}^{\text {(l+1 }}$ should look like: we let

$$
\mathscr{F}^{(r+1}=\left\langle\left\langle\mathscr{F}^{(x)} \cup\left\{Z_{f}: f \in{ }^{\omega} \omega \text { and } \forall n f(n) \leq n\right\}\right\rangle\right\rangle
$$

where $Z_{f}=\omega-\bigcup_{i<(i)} D_{i, f(i)}$. 
To define $\mathscr{F}_{\beta, n}^{\alpha+1}$ for $\beta<\mu$ we pick one $\zeta$ from $J_{\alpha}$, let $J_{\alpha+1}=J_{\alpha}-\{\zeta\}$ and we put

$$
\mathscr{F}_{\beta, n}^{(\alpha+1}=\left\langle\left\langle\mathscr{F}_{\beta, n}^{\alpha} \cup\left\{A_{\xi, k}: n \in B_{\zeta, k}\right\}\right\rangle\right\rangle \text {. }
$$

Again only (v) and (vi) need verification: we begin with (v).

If $\beta<\mu$ and $n<\omega$ then $\mathscr{A}_{\alpha+1}$ is independent with respect to $\mathscr{F}_{\beta, n}^{\alpha+1}$ by (2.4). This leaves $\mathscr{F}^{\alpha+1}$ : let $H \in \mathscr{F}^{\alpha}$, let $K$ be a finite set of functions, let $\varphi \in \operatorname{Fn}\left(I_{\alpha+1}, \omega\right)$, and let $\gamma<\mu$. By our assumptions on $\mathscr{D}_{\alpha}$ and $\mathscr{F}^{(\alpha}$ we can assume that $H \cap D_{n}=\varnothing$ if $n \leq|K|+1$. Now by (1) we can take some $i \in L_{\gamma}$ such that $H \in \mathscr{F}_{\gamma, i}{ }^{k}$, we may also take an $n$ such that $D_{n} \in \mathscr{F}_{\gamma, i}^{\alpha}$; it follows that $n>|K|+1$, so that for some $k \leq n$ we have $D_{n, k} \subseteq \bigcap_{f \in K} Z_{f}$. Because $\mathscr{A}_{\alpha}$ is independent with respect to $\mathscr{F}_{\gamma, i}{ }^{k}$ and $\xi \notin I_{\alpha+1}$ it now follows easily that $H \cap \bigcap_{f \in K} Z_{f} \cap A(\varphi)$ is nonempty.

Next we do (vi). Again if $\beta<\mu$ there is no problem. We simply note that for $\gamma<\beta \quad \mathscr{G}_{\beta, \gamma, n}^{(\alpha+1}=\left\langle\left\langle\mathscr{G}_{\beta, \gamma, n}^{\mathrm{\alpha}} \cup\left\{B_{\zeta, k}\right\}\right\rangle\right\rangle$ for an appropriate $k$.

For $\mathscr{F}^{\alpha+1}$ let $H$ and $K$ be as above, let $\tau \in \operatorname{Fn}\left(J_{\alpha+1}, \omega\right)$, and let $\gamma<\mu$. Take a $\beta$ with $\gamma<\beta<\mu$. The argument given above provides us with $m$, $k$ and $n$ such that $D_{m, k} \subseteq \bigcap_{f \in K} Z_{f}$ and $H \cap D_{m} \in \mathscr{F}_{\beta, n}$. Now $\left\{i: D_{m, k} \in\right.$ $\left.\mathscr{F}_{\gamma, i}{ }^{\alpha+1}\right\}=\left\{i: D_{m} \in \mathscr{F}_{\gamma, i}{ }^{\alpha+1}\right.$ and $\left.A_{\xi, k} \in \mathscr{F}_{\gamma, i}^{(x+1}\right\}=\left\{i \in B_{\zeta, k}: D_{m} \in \mathscr{F}_{\gamma, i}{ }^{\alpha}\right\}=$ $E\left(D_{m}, \gamma\right) \cap B_{\zeta, k}$. Using the independence of $\mathscr{B}_{\alpha}$ with respect to $\mathscr{G}_{\beta, \gamma, n}^{\left(\gamma^{\prime},\right.}$ it now follows easily that $\left\{i: H \cap \bigcap_{f \in K} Z_{f} \cap B(\tau) \in \mathscr{F}_{\gamma, i}{ }^{(x+1}\right\} \neq \varnothing$.

The case when (2) holds is somewhat easier. We follow the strategy of the odd step: let $M=\bigcup\left\{A_{\gamma, k}: k \in L_{\gamma}\right\}$. One readily checks that-in the notation of that step- $L_{\gamma}=\{k:\langle\gamma, k\rangle \in C\}$, so that $\mathscr{F}^{\alpha} \in C_{1}$ and hence $\omega-M \in \mathscr{F}^{(\alpha+1}$.

This completes the construction.

\section{ACKNOWLEDGMENT}

The author is indebted to Klaas Pieter Hart and Petr Simon for their help in the preparation of this paper.

\section{REFERENCES}

[BB] L. Bukovský and E. Butkovičová, Ultrafilter with $\aleph_{0}$ predecessors in Rudin-Frolik order, Comm. Math. Univ. Carolinae 22 (1981), 429-447.

[vD] E. K. van Douwen, $A$ c-chain of copies of $\beta \omega$, Coll. Math. Soc. János Bolyai, no. 41, Topology and Applications, Eger (1983), 261-267.

[EKa] R. Engelking and M. Karlowicz, Some theorems of set theory and their topological consequences, Fund. Math. 57 (1965), 275-285.

[F1] Z. Frolik, Sums of ultrafilters, Bull. Amer. Math. Soc. 73 (1967), 87-91.

[F2] Z. Frolik, Homogeneity problems for extremally disconnected spaces, Comm. Math. Univ. Carolinae 8 (1967), 757-763.

[K] K. Kunen, Ultrafilters and independent sets, Trans. Amer. Math. Soc. 172 (1972), 299-306.

[R] M. E. Rudin, Partial orders of the types in $\beta \mathbb{N}$, Trans. Amer. Math. Soc. 155 (1971), 353-362.

Mathematical Institute, Slovak Academy of Sciences, Mú SAV, Jesenná 5, 04154 Košice, Czechoslovakia 